\title{
BMJ Open Efficacy of vibrotactile device DentalVibe in reducing injection pain and anxiety during local anaesthesia in paediatric dental patients: a study protocol for a randomised controlled clinical trial
}

\author{
Elitsa Veneva, ${ }^{\oplus 1}$ Radka Cholakova, ${ }^{2}$ Ralitsa Raycheva, ${ }^{\circ}$ Ani Belcheva ${ }^{1}$
}

To cite: Veneva E, Cholakova $\mathrm{R}$, Raycheva $\mathrm{R}$, et al. Efficacy of vibrotactile device DentalVibe in reducing injection pain and anxiety during local anaesthesia in paediatric dental patients: a study protocol for a randomised controlled clinical trial. BMJ Open 2019;9:e029460. doi:10.1136/ bmjopen-2019-029460

\section{- Prepublication history and} additional material for this paper are available online. To view these files, please visit the journal online (http://dx.doi org/10.1136/bmjopen-2019029460).

Received 27 January 2019 Revised 24 May 2019 Accepted 28 May 2019
Check for updates

(C) Author(s) (or their employer(s)) 2019. Re-use permitted under CC BY-NC. No commercial re-use. See rights and permissions. Published by BMJ.

For numbered affiliations see end of article.

Correspondence to Dr Elitsa Veneva; elitza.veneva@gmail.com

\section{ABSTRACT}

Introduction A current non-pharmacological mean for attaining painless local anaesthesia $(\mathrm{LA})$ is presented by vibrotactile devices. Their concept is to reduce injection pain due to distraction by applying physical stimuli which interfere with pain signals. The aim of this study is to determine the efficacy of the DentalVibe (DV) device in reducing pain and anxiety associated with $L A$ in paediatric patients.

Methods and analysis The proposed study is a randomised controlled clinical trial with split-mouth design Included are positive patients aged 8-12 years, requiring buccal infiltration for extraction of two bilateral primary maxillary molars. After dental fear measurement, eligible patients undergo two single-visit treatments with DV device allocated to either first or second LA via computergenerated randomisation sequence. Outcome measures will be self-reported pain felt during LA on Visual Analogue Scale; self-reported anxiety on Facial Image Scale; painrelated behaviour according to Faces, Legs, Activity, Cry, Consolability Scale; heart rate; patient preference to LA technique. Data will be analysed with intention-to-treat concept by Student's t-test for paired samples, Wilcoxon signed-rank test, $p<0.05$. Pretest on 20 subjects resulted in $\mathrm{n}=41$ patients sample size.

Ethics and dissemination This study protocol has been approved by the Committee for Scientific Research Ethics, Medical University - Plovdiv, Bulgaria (Reference number P-8604, Protocol of approval No. 6/23.11.2017) and registered on a publicly accessible database. This research received institutional funding from the Medical University - Plovdiv, Bulgaria, under project SPD-03/2017. Findings will be reported in scientific publications and at research conferences, and in project summary papers for participants.

Trial registration number NCT03445182; Pre-results.

\section{INTRODUCTION}

Background and rationale

Painless treatment is an integral element of quality paediatric dental care. Fear associated
Strengths and limitations of this study

- Vibrotactile devices such as DentalVibe (DV) present a new non-pharmacological method that could allow the clinician a treatment approach, associated with reduced injection pain, essential for managing anxiety in paediatric patients.

- This randomised controlled trial is a well-powered, one-centre experimental study with split-mouth design.

- The clinical adequacy of DV-assisted injection for achieving painless infiltration of local anaesthesia $(\mathrm{LA})$ is investigated in a complex manner with both subjective and objective measurements of pain, anxiety, dental fear and heart rate in subjects from a narrow age group without previous experience with LA.

- This study involves only one operator and one primary investigator, both of female gender, reducing interindividual variability from the estimates of the treatment effect.

- Disadvantages of this trial include the need to find positive patients with symmetrical and similar conditions, and many patients are not eligible, as well as the open-label design of the study due to the nature of the investigated device.

with seeing and experiencing needle penetration, as well as sensation of swelling soft tissues, is the most common factor causing children and dental clinicians to experience anxiety regarding the use of infiltration local anaesthesia (LA). Recent progress in the field of dental pain management has led to development of newer delivery devices and also modification in injection techniques. Their aim is to allow the clinician a treatment approach, associated with reduced injection pain, essential for managing anxiety in paediatric patients. 
The gate control theory of pain by Melzack ${ }^{1}$ is a widely accepted concept of pain perception. In recent years, several innovative dental appliances have been developed on its basis-Accupal, DentalVibe (DV), Vibraject and others. ${ }^{2}$ Their concept is to reduce the pain of needle injection by applying pressure, vibration, microoscillations or a combination of them. The applied physical stimuli are hypothesised to modify or interfere with pain signals by closing the neural gate of cerebral cortex, aimed to decrease the pain perception due to distraction.

DentalVibe Gen4 Comfort Injection system is designed to reduce injection pain by applying pressure and vibration at the injection site. The number of conducted clinical trials regarding its efficacy and acceptance in paediatric patients is insufficient to validate the method as effective and applicable in this age group. Exploring this new non-pharmacological mean for attaining painless LA will help improve the quality of care in paediatric dentistry by evaluating its clinical adequacy.

\section{Objectives}

The intention of the technique of DV is to reduce pain sensation in that large percentage of patients who may experience unpleasant sensations during infiltration of LA. We hypothesised that when pressure and vibration are applied at the injection site, a decrease in pain perception could be expected due to distraction. The child's physical, psychological and cognitive development may affect the experience of pain and anxiety during infiltration of LA. Therefore, a thorough investigation of the method's clinical adequacy should be carried out in a complex manner to validate it as applicable in paediatric patients.

The aim of this study was efficacy approbation and patient acceptance evaluation of DV-assisted injection in reducing pain and anxiety associated with LA in paediatric dental patients.

After dental fear assessment, the main objectives are to compare pain felt during LA with DV-assisted injection compared with traditional infiltration-self-reported pain and observed pain-related behaviour. The second objective includes evaluation of self-reported anxiety during LA in both groups, as well as heart rate dynamics during the procedures. As in this split-mouth trial every patient receives both interventions, patient's preference to LA method will be estimated after completion of treatment.

\section{Trial design}

The trial to be conducted is a randomised controlled clinical cross-over experimental study with split-mouth design. The within-subject design randomly allocates experimental and control interventions to different areas in the oral cavity (teeth, surfaces, arches, quadrants) and has the advantage of reducing outcome variability estimation since each patient is in his/her own control, leading to potential increase in statistical power. A disadvantage of the split-mouth design is the need of including patients with symmetrical and similar dental defects/conditions,

\begin{tabular}{|c|c|c|c|c|c|c|c|}
\hline & \multicolumn{7}{|c|}{ STUDY PERIOD } \\
\hline \multirow[b]{2}{*}{ TIMEPOINT* } & \multirow{2}{*}{$\begin{array}{c}\text { Enrolment } \\
t_{1}\end{array}$} & \multirow{2}{*}{$\begin{array}{c}\text { Allocation } \\
0\end{array}$} & \multicolumn{5}{|c|}{ Post-allocation } \\
\hline & & & $t_{1}$ & $t_{2}$ & $t_{3}$ & $t_{4}$ & $t_{5}$ \\
\hline \multicolumn{8}{|l|}{ ENROLMENT: } \\
\hline Eligibility screen & $\mathrm{x}$ & & & & & & \\
\hline Informed consent & $x$ & & & & & & \\
\hline Allocation & & $\mathrm{x}$ & & & & & \\
\hline \multicolumn{8}{|l|}{ INTERVENTIONS: } \\
\hline $\begin{array}{r}\text { Traditional local } \\
\text { anaesthesia }\end{array}$ & & & & $x$ & & & \\
\hline $\begin{array}{r}D V \text {-assisted local } \\
\text { anaesthesia }\end{array}$ & & & & $x$ & & & \\
\hline \multicolumn{8}{|l|}{ ASSESSMENTS: } \\
\hline \multirow{2}{*}{$\begin{array}{r}\text { Dental Fear } \\
\text { Pain felt during } \\
\text { treatment }\end{array}$} & & & $x$ & & & & \\
\hline & & & & & $x$ & & \\
\hline $\begin{array}{r}\text { Anxiety felt during } \\
\text { treatment }\end{array}$ & & & & & $\mathrm{x}$ & & \\
\hline $\begin{array}{r}\text { Pain-related } \\
\text { behaviour }\end{array}$ & & & & $x$ & & & \\
\hline \multicolumn{8}{|l|}{ Heart rate } \\
\hline Patient preference & & & & & & & $x$ \\
\hline
\end{tabular}

Figure 1 Schedule of enrolment, interventions and assessments of treatments in this randomised controlled trial. ${ }^{*}$ Post-allocation time frame: $t_{1}$ - before start of treatment; $t_{2}-$ during local anaesthetic infiltration; $t_{3}$-immediately after local anaesthesia; $t_{4}$ - end of treatment, before leaving the dental chair; $t_{5}-1$ week after second visit. DV, DentalVibe.

and many patients are not eligible. Figure 1 summarises the enrolment, intervention and assessment schedule, all of which are in accordance with the Standard Protocol Items: Recommendations for Interventional Trials recommendations. ${ }^{3}$

This is an open-label trial, that is, unmasked, where patients, operator and outcomes assessor are not blinded for the study due to the nature of the investigated device. Experimental procedure in this study consists of a DV-assisted local anaesthetic injection prior to extraction of a primary upper jaw molar, whereas control manipulation consists of conventional anaesthetic injection prior to extraction of a contralateral primary upper jaw molar of the same patient.

The required number of patients is calculated on the basis of a microsample at an accepted level of significance $(\mathrm{p}<0.05)$ and a maximum permissible error $\alpha<0.05$ and $\beta<0.2$. Intention-to-treat concept is chosen as statistical approach for data analysis.

\section{METHODS AND ANALYSIS}

\section{Study setting}

The setting of this trial is the Faculty of Dental Medicine, Medical University-Plovdiv, Bulgaria. Eligible patients are selected from the visitors of the paediatric dental clinic of the Department of Paediatric Dentistry and treated in the Department of Oral Surgery of the aforementioned. 


\section{Eligibility}

Inclusion criteria

1. Participants in the study are children 8-12 years old.

2. Children identified as positive or definitely positive through Frankl Behavioural Rating Scale.

3. Children who are not considered medically compromised or medically complex patients. The absence of disease is confirmed by anamnestic interview with a parent or a caregiver of the child and excludes general acute or chronic disease, cognitive impairment.

4. Children without previous experience with LA, requiring LA infiltration for extraction of two primary upper jaw molars bilaterally. Indications for extraction of primary molars: over-retention-in case of delayed physiological change and tendency of eruption of the permanent tooth; orthodontic reasons-tooth removed to prevent or correct malocclusion; advanced root resorption and imminent physiological tooth replacement; teeth with a severely damaged clinical crown (from trauma or caries and its complications); teeth with failed pulpotomy, acute or chronic diffuse periodontitis, endangering the germ of the permanent tooth.

5. Obtained written informed consent from parents or caregivers, as well as obtained verbal informed consent from the child to participate in the study, in which procedures are explained in age-appropriate manner.

\section{Exclusion criteria}

1. Patients who are undergoing therapy with neurological, sedative, analgesic and/or anti-inflammatory drugs 7 days prior to treatment.

2. Children who are first-time ever dental patients.

3. Patients with bisulfite sensitivity or allergy to local anaesthetics of the amide group.

\section{Interventions}

The injection technique used for both control and experimental manipulation is conventional buccal infiltration in the posterior maxillary region. A 27 gauge $16 \mathrm{~mm}$ needle is inserted in the mucobuccal fold above the tooth to be anaesthetised. Local anaesthetic infiltration speed is $1 \mathrm{~mL} / \mathrm{min}$.

Local anaesthetic agent is chosen to be Ubistesin- $4 \%$ Articaine with epinephrine 1:200000 1.7 mL. For the experimental arm, the injection is assisted by the DV device. The tip of the DV device is placed in the mucobuccal fold above the tooth to be anaesthetised. The device is activated for $5 \mathrm{~s}$. A 27 gauge $16 \mathrm{~mm}$ short needle is inserted as close as possible to the inner side of the prong while the vibration is still on. Local anaesthetic infiltration speed is $1 \mathrm{~mL} / \mathrm{min}$. After injection, the needle is withdrawn and vibration continues for another $5 \mathrm{~s}$. Arms and assigned interventions in the randomised controlled trial (RCT) are shown in table 1.
Table 1 Arms and assigned interventions of the cross-over randomised trial

\begin{tabular}{|c|c|}
\hline Arms & Assigned interventions \\
\hline $\begin{array}{l}\text { Active comparator: } \\
\text { Traditional LA } \\
\text { LA with conventional } \\
\text { syringe }\end{array}$ & $\begin{array}{l}\text { Procedure: LA with conventional } \\
\text { syringe. } \\
\text { Buccal infiltration in posterior } \\
\text { maxillary region with traditional } \\
\text { technique. A } 27 \text { gauge } 16 \mathrm{~mm} \\
\text { short needle is inserted in the } \\
\text { mucobuccal fold above the } \\
\text { tooth to be anaesthetised. Local } \\
\text { anaesthetic infiltration speed is } \\
1 \mathrm{~mL} / \mathrm{min} \text {. } \\
\text { Drug: Local anaesthetic- } \\
\text { Ubistesin- } 4 \% \text { Articaine with } \\
\text { epinephrine } 1: 2000001.7 \mathrm{~mL}\end{array}$ \\
\hline $\begin{array}{l}\text { Active comparator: DV- } \\
\text { assisted LA } \\
\text { LA with conventional } \\
\text { syringe+DV }\end{array}$ & $\begin{array}{l}\text { Device: LA with conventional } \\
\text { syringe+DV } \\
\text { Buccal infiltration in posterior } \\
\text { maxillary region. The tip of } \\
\text { the DV device is placed in the } \\
\text { mucobuccal fold above the tooth } \\
\text { to be anaesthetised. The device is } \\
\text { activated for } 5 \mathrm{~s} \text {. A } 27 \text { gauge } 16 \mathrm{~mm} \\
\text { short needle is inserted as close } \\
\text { as possible to the inner side of the } \\
\text { prong while the vibration is still on. } \\
\text { Local anaesthetic infiltration speed } \\
\text { is } 1 \mathrm{~mL} / \mathrm{min} \text {. After injection, the } \\
\text { needle is withdrawn and vibration } \\
\text { continues for another } 5 \mathrm{~s} \text {. } \\
\text { Drug: Local anaesthetic- } \\
\text { Ubistesin- } 4 \% \text { Articaine with } \\
\text { epinephrine } 1: 2000001.7 \mathrm{~mL}\end{array}$ \\
\hline
\end{tabular}

DV, DentalVibe; LA, local anaesthesia.

\section{Clinical protocol}

Eligible patients undergo two single-visit treatments after measurement of dental fear prior to each visit. LA is delivered through buccal infiltration with conventional syringe, whereas DV device is allocated to either first or second LA procedure via computer-generated permuted-block randomisation sequence. Patients in the control group receive traditional LA at the first visit and DV injection at the second, while for children in the experimental group, the DV device will be introduced as first method of LA. No topical anaesthetic is to be used prior to injection in both groups as not to create bias in results.

Workflow:

1. Parents or caregivers are informed about LA and the DV-assisted method of infiltration. They get acquainted with the nature of the research being conducted in to prepare their children for the dental treatment (see online supplementary data file S1-'Information leaflet'). The parent or caregiver signs informed consent (see online supplementary data file S2-'Informed consent'). Verbal assent as the child's affirmative agreement to participate in research is received. 
2. Patients are asked to complete Children's Fear Survey Schedule-Dental Subscale (CFSS-DS) questionnaire.

3. Pulse oximeter is connected to patient's index finger. Start of heart rate monitoring and recording $-5 \mathrm{~min}$ prior to treatment. Time frame: until end of treatment before leaving the dental chair.

4. Prior to local anaesthetic injection, the 'tell-show-do' technique is used in both groups to present the upcoming treatment, as well as age-specific terminology to explain manipulations. LA is presented as 'putting the tooth to sleep'; the anaesthetic solution is called 'sleepy juice', the needle-a 'straw, from which the tooth drinks the sleepy juice', the DV device-the 'little crocodile'.

5. Local anaesthetic delivery is performed according to assigned intervention. The outcomes assessor monitors the patient during the procedure and registers pain-related behaviour using Faces, Legs, Activity, Cry, Consolability (FLACC) Behavioural Pain Rating Scale. ${ }^{4}$

6. Immediately after local anaesthetic delivery, the patient is asked to rate their pain perception on Visual Analogue Scale (VAS) and their anxiety during injection on Facial Image Scale (FIS).$^{5}$

7. Follows extraction of primary upper molar.

8. One week after the second dental visit, the patient is reached by a phone call and asked for his/her preference for LA method.

\section{Outcomes}

\section{Primary outcome measures}

The primary outcome measure will be pain felt during injection self-reported by the patient immediately after LA infiltration using a VAS, containing a combination of Numeric Rating Scale (0-10, where 0 means no pain, 10 means worst possible pain) and Wong-Baker Faces Pain Scale, including pictures of facial expressions with correlating numbers of $0-10$ ( 0 being 'no hurt' and 10 being 'hurts worst'). The combination allows children to pick a facial expression that corresponds with their pain and see a number that matches it.

\section{Secondary outcome measures}

Assessment of the following secondary outcomes will be performed: (1) pain-related behaviour during LA, evaluated by the outcomes assessor using the FLACC Behavioural Pain Rating Scale. The FLACC Behavioural Pain Rating Scale has five criteria, which are each assigned a score of 0,1 or 2 . Total score of scale is summed in range from 0 to 10 , where $0=$ relaxed and comfortable, 1-3=mild discomfort, 4-6=moderate pain, 7-10=severe pain; (2) self-reported anxiety during injection evaluated on FIS which comprises a row of five faces from very unhappy (score 5) to very happy (score 1) (time frame: immediately after local anaesthetic delivery); (3) heart rate dynamics during the experiment, registered via pulse oximeter. Patient's left index finger is connected to a portable recording pulse oximeter in the waiting room, at least $5 \mathrm{~min}$ before LA procedure until the end of treatment; (4) assessment of self-reported dental fear on CFSS-DS questionnaire-performed prior to both visits. The CFSS-DS questionnaire consists of 15 items related to different aspects of dental treatment, including fear of injections. Total score: $15-75$; (5) patient's preference to LA method. One week after the second dental visit, the patient is reached by a phone call and asked: "If we have to put another tooth of yours to sleep, which method would you prefer to put your tooth to sleep-with the vibrating device (the little crocodile) or without it?"

\section{Participant's timeline}

Each eligible patient undergoes two single-visit treatments for extraction of two primary upper jaw molars, receiving traditional injection prior to extraction of one tooth and DV-assisted injection at the other visit prior to extraction of the homologous contralateral tooth. LA and extraction procedure in both visits are performed by the same operator of female gender for all participants, who had 2 months experience with the device. Outcomes are registered by the primary investigator, who is not the operator, in a clinical file. An interval of 7-21 days is allowed between one procedure and the other. The protocol to be used in the first procedure is randomly selected using a computer-generated list linked to sequence of enrolment in the trial.

\section{Sample size calculation}

Given the lack of fully comparable research and the unknown population SD, we conducted a pretest with 20 subjects and considered the behaviour of this subgroup as population estimate. To estimate sample size for the primary outcome-self-reported pain felt during injection, according to the VAS-we applied a t-test for paired groups ( $G^{*}$ Power software V.3.1, ${ }^{6}$ since we have two groups (primary molar in the right and left quadrants of upper jaw) on the same patient.

The effect size was determined using the formula

$$
E S=\frac{\text { Control }- \text { Treated }}{\text { SDpooled }}=\frac{2.33-0.33}{3.25}=0.62,
$$

where SD is the pooled SD, an average of the SD of the experimental and control groups. The error was set at $5 \%$ and the power test at $95 \%$. According to the calculation, a sample of 37 patients will be necessary to detect differences in pain. Since dropouts are unavoidable when collecting follow-up data, this number needs to be adjusted for the estimated dropout rate. During the pretest, we had a dropout rate of $5 \%$ for collecting the follow-up data, and if we anticipated a higher dropout rate for this study, we conservatively allowed for a $10 \%$ dropout rate. Adjusting the sample size for this dropout rate results in a sample of 41 patients needing to be recruited.

\section{Recruitment}

The clinical trial is currently recruiting participants. Patient recruitment started in April 2018. Estimated primary study completion date is March 2019. The enrolment capacity was estimated to be five patients/month. 


\section{Participating centres}

Eligible patients are selected from the visitors of the paediatric dental clinic of the Department of Paediatric Dentistry, Faculty of Dental Medicine - Medical University of Plovdiv, Bulgaria, and treated in the Department of Oral Surgery of the aforementioned.

\section{Assignment of interventions}

Sequence generation

A computer-generated, permuted-block randomisation sequence for allocation of first procedure is to be prepared. Patients are to be randomised to treatment allocation according to number of enrolment in the trial. In this split-mouth RCT, every patient will receive both procedures. The patient will be randomised to receive (1) at the first visit, LA with conventional syringe and, at the second visit,LA with conventional syringe, assisted by DV or (2) at the first visit, DV-assisted LA and, at second one, traditional infiltration anaesthesia.

\section{Allocation concealment mechanism and implementation}

Randomisation is based on treatment allocation sequence number. The first procedure to be received by each patient is linked to the number of enrolment in the study in a Microsoft Excel table (where (1) is local anaesthesia with conventional syringe, and (2) is local anaesthesia with conventional syringe, assisted by the DentalVibe device). Allocation sequence will be generated before the start of the patient enrolment by the statistician. The operator will obtain each randomisation allocation via a sequentially numbered opaque sealed envelope prior to treatment, enabling the sequence to be concealed until the intervention is assigned. Patients will be enrolled by the primary investigator.

\section{Blinding}

The current study is designed as an open-label trial. Patients and outcomes assessor cannot be blinded due to the nature of the investigated device. The operator will get acquainted with the procedure to be performed prior to treatment session. The clinicians involved in this study as operator and outcomes assessor are selected to be the only ones performing the manipulations in order to prevent bias.

\section{Data collection, confidentiality, storage and monitoring of study documents}

According to the regulations of the Personal Data Protection Act, the collected paper forms will be stored in a secure manner in the Department of Paediatric Dentistry, Faculty of Dental Medicine, Medical University - Plovdiv, Bulgaria. Outcomes will be transferred electronically by the primary investigator after transmission of paper forms. Clinical research files will be stored in a locked, secured office. Data will be electronically stored on a double password-protected computer. Only the primary investigator and the statistician will have access to the final data set. The trial will be monitored by the research monitoring officer of Medical University - Plovdiv, verifying that the study is conducted in accordance with the Good Clinical Practice guidelines.

\section{STATISTICAL METHODS}

The unit of analysis will be the tooth for the split-mouth RCT (two primary molars belonging to the same dental arch treated per patient). The data will be recorded and analysed using SPSS 22.0. All data will be analysed using an intention-to-treat analysis. ${ }^{7}$ Descriptive statistics will be calculated. Discrete variables will be summarised by frequencies or proportions. Continuous variables will be reported as means and SE or medians and range (depending on the distribution of the variables). Data will be checked for baseline differences between the treatment arms. If baseline differences do occur for any of the variables, they will be added to subsequent models to compensate for those differences using an analysis of covariance approach.

We will compare pain mean scores according to the VAS, containing numerical symbols. We will report the mean differences between groups and the associated 95\% CI. For the split-mouth RCT, with each patient being in his or her own control, our statistical analysis will take into account the paired nature of data and the results will be analysed by Student's t-test for paired samples or Wilcoxon signed-rank tests, if the data are not normally distributed.

\section{Patient and public involvement}

The development of this current research question and outcome measures was informed by the priorities of patients' parents or caregivers in a way that this non-pharmacological approach towards LA may be suitable for children who have no experience with infiltrative analgesia and might benefit higher acceptance of treatment. Patients or their parents/caregivers were not involved during the phase of study design; however, their concerns and questions will be addressed during patient recruitment and study implementation. The results will be disseminated to study participants through email and routine follow-up dental check-up. Indicators of intervention burden will be partially patient self-reported, such as subjective pain and anxiety during the procedure, as well as patient's preference to LA method, while the other outcomes will be assessed by the research investigators.

\section{ETHICS AND DISSEMINATION}

The clinical trial will be carried out in line with the principles of the Declaration of Helsinki and according to the Clinical Trials Directive 2001/20/EC of the European Parliament on the approximation of the laws, regulations and administrative provisions of the Member States relating to the implementation of Good Clinical Practices in the conduct of clinical trials on medicinal products for human use. 


\section{Research ethics approval}

This study has been approved by the Committee for Scientific Research Ethics, Medical University - Plovdiv, Bulgaria (Reference number P-8604, Protocol amendment number: 01, Protocol of approval No. 6/23.11.2017) and registered on a publicly accessible database ClinicalTrials. gov (Registration number: NCT03445182). The protocol of the study and the written information leaflets and informed consent documents are approved by the ethics committee. Should there be any changes in the aforementioned, they will be consulted with the committee.

\section{Consent}

Parents or caregivers will be given written informed consent and information leaflets by the primary investigator in person. Verbal assent as a child's affirmative agreement to participate in research will be sought.

\section{Confidentiality}

People with direct access to the data will take all necessary precautions to maintain confidentiality. All data collected during the study will be rendered anonymous. Only initials and inclusion number will be registered.

\section{Dissemination policy}

The results of the study will be released to dental medicine specialists and scientific community no later than 1 year after completion of the trial through presentation at scientific conferences and publication in peer-reviewed journals. The principal investigator $(\mathrm{EV})$, the statistician $(\mathrm{RR})$, the scientific expert (AB) and the operator (RC) will write the first draft of the manuscript without use of professional writers.

\section{DISCUSSION}

The child's physical, psychological and cognitive development may affect the experience of pain and anxiety during LA. Pain measurement complexity in children is exacerbated by the fact that it is difficult to distinguish between behaviour resulting purely from pain and behaviour resulting from fear and a mixture of other factors. $^{89}$

Since paediatric patients' subjective evaluation of pain may affect the reliability of the results, in this study the efficacy of the DV device is investigated in a complex manner with both subjective and objective measurements of dental fear, anxiety, pain and heart rate. The subjects in this study have no previous experience with LA. Dental fear is measured prior to each visit, as a crucial factor correlating with reported pain and previous memories after being subjected to it once. During LA procedure, the outcomes assessor records pain-related behaviour. After anaesthesia completion, the patient reports intraoperative distress on FIS and subjective pain on VAS. Interinvestigator differences are eliminated by inclusion of only one operator, performing the anaesthetic injection, and one outcomes assessor-both of female gender, since a study on the effect of operator and subject gender on injection pain by Perry et al concluded that gender had a statistically significant effect for solution deposition pain when a male operator administered the injection to female subjects. ${ }^{10}$ The following precautions are taken in account to minimise variables in results-eligible patients are selected from a narrow age group (10-12 years), classified as positive or definitely positive through Frankl Behavioural Rating Scale; indications for LA are limited to extraction of primary upper jaw molars.

Discomfort from LA injection is related to both needle penetration and tension created by tissue expansion as the anaesthetic is infiltrated. The vibration of the DV is found to relieve pain of the needle prick, but may also reduce infiltration pain by physically facilitating the distribution of the anaesthetic fluid. Although it is hypothesised that it might promote anaesthetic effect induction, a study by Shaefer $e t$ al found that the use of DV did not influence the time for complete anaesthesia $(p>0.05) .{ }^{11}$ In research, there are few studies regarding the use of vibrotactile devices in paediatric patients. ${ }^{12-16}$ The results of some studies state that DV can alleviate pain associated with dental injections in paediatric patients. ${ }^{12} 14$

This RCT is a well-powered, one-centre split-mouth experimental study. Assessment of subjective and objective pain sensation and anxiety during treatment of similar cases should help estimate the clinical adequacy of the DV-assisted infiltration method by finding out if pain-free LA can subsequently be performed.

\section{Trial status}

This trial is currently recruiting patients. Patient recruitment started in April 2018, and by January 2019, 28 patients are enrolled. Pretest on 20 subjects resulted in $\mathrm{n}=41$ patients needing to be recruited. Outcome results will be updated in ClinicalTrials.gov after completion of the study, estimated due March 2019.

\section{Author affiliations}

${ }^{1}$ Department of Pediatric Dentistry, Faculty of Dental Medicine, Medical University Plovdiv, Plovdiv, Bulgaria

${ }^{2}$ Department of Oral Surgery, Faculty of Dental Medicine, Medical University -

Plovdiv, Plovdiv, Bulgaria

${ }^{3}$ Department of Social Medicine and Public Health, Faculty of Public Health, Medical University - Plovdiv, Plovdiv, Bulgaria

Acknowledgements We would like to show our gratitude to the Department of Oral Surgery for their support in facilitating this project. We are also thankful to all the parents and children for participating in the trial.

Contributors We declare that all authors have made substantial contributions. $A B$ and $E V$ conceived the ideas. $R C$ was the primary operator, while $E V$ was the outcomes assessor and data collector. Writing was led by EV and RR. Design and protocol for this study was developed by EV, AB and RR. Data curation by R.R. Project administration was led by EV.

Funding This research received institutional funding from the Medical University Plovdiv, Bulgaria, under 'Start of the doctoral programs' approved research project No. SPD-03/2017. The funder has no role in study design; collection, management, analysis and interpretation of data; writing of the report; and the decision to submit the report for publication.

Competing interests None declared.

Patient consent for publication Not required. 
Provenance and peer review Not commissioned; externally peer reviewed.

Open access This is an open access article distributed in accordance with the Creative Commons Attribution Non Commercial (CC BY-NC 4.0) license, which permits others to distribute, remix, adapt, build upon this work non-commercially, and license their derivative works on different terms, provided the original work is properly cited, appropriate credit is given, any changes made indicated, and the use is non-commercial. See: http://creativecommons.org/licenses/by-nc/4.0/.

\section{REFERENCES}

1. Melzack R. Gate control theory. Pain Forum 1996;5:128-38.

2. Sriram G. Advances in Local Anaesthesia : A Paediatric overview. Indian J Dent Adv 2014;6:1605-7.

3. Chan AW, Tetzlaff JM, Altman DG, et al. SPIRIT 2013 statement: defining standard protocol items for clinical trials. Ann Intern Med 2013;158:200-7

4. Merkel SI, Voepel-Lewis T, Shayevitz JR, et al. The FLACC: a behavioral scale for scoring postoperative pain in young children. Pediatr Nurs 1997;23:293-7.

5. Buchanan $\mathrm{H}$, Niven N. Validation of a Facial Image Scale to assess child dental anxiety. Int J Paediatr Dent 2002;12:47-52.

6. Faul F, Erdfelder E, Lang AG, et al. G*Power 3: a flexible statistical power analysis program for the social, behavioral, and biomedical sciences. Behav Res Methods 2007;39:175-91.

7. White IR, Horton NJ, Carpenter J, et al. Strategy for intention to treat analysis in randomised trials with missing outcome data. BMJ 2011;342:d40.
8. Versloot J, Veerkamp JS, Hoogstraten J. Assessment of pain by the child, dentist, and independent observers. Pediatr Dent 2004;26:445-9.

9. Beyer JE, McGrath PJ, Berde CB. Discordance between self-report and behavioral pain measures in children aged 3-7 years after surgery. J Pain Symptom Manage 1990;5:350-6.

10. Perry S, Drum M, Reader A, et al. Effect of operator and subject gender on injection pain: a randomized double-blind study. J Endod 2015;41:141-5.

11. Shaefer JR, Lee SJ, Anderson NK. A vibration device to control injection discomfort. Compend Contin Educ Dent 2017;38:e5-e8.

12. Ching D, Lcy FM. Effect of the DentalVibe injection system on pain during local anesthesia injections in adolescent patients. 2014;36:51-5.

13. Elbay M, Sermet Elbay Ü, Yıldırım S, et al. Comparison of injection pain caused by the DentalVibe Injection System versus a traditional syringe for inferior alveolar nerve block anaesthesia in paediatric patients. Eur J Paediatr Dent 2015;16:123-8.

14. Dak-Albab R, Al-Monaqel MB, Koshha R, et al. A comparison between the effectiveness of vibration with Dentalvibe and benzocaine gel in relieving pain associated with mandibular injection: a randomized clinical trial. Anaesthesia, Pain Intensive Care 2016;20:43-9.

15. Shilpapriya M, Jayanthi M, Reddy VN, et al. Effectiveness of new vibration delivery system on pain associated with injection of local anesthesia in children. J Indian Soc Pedod Prev Dent 2015;33:173-6.

16. Chaudhry K, Shishodia M, Singh C, et al. Comparative evaluation of pain perception by vibrating needle (Vibraject TM) and conventional syringe anesthesia during various dental procedures in pediatric patients : a short study. Int Dent Med J Adv Res - Vol 2015;1:1-5 